\title{
HYBRID NEURO-WAVELET MODEL FOR SHORT TERM LOAD FORECASTING
}

\author{
Ravindra M. Gimonkar ${ }^{1,}$ D.A.Kapgate ${ }^{2}$ \\ ${ }^{1}$ Associate Prof, Department of Electrical Engg., ${ }^{2}$ Asst. Prof. Department of Electronics \& Communication Engg \\ ${ }^{1}$ YCCE Nagpur, Autonomous Institute, RTM Nagpur University, Nagpur, Maharashtra State, India \\ ${ }^{2}$ PCE Nagpur, RTM Nagpur University, Nagpur, Maharashtra State, India
}

\begin{abstract}
Accuracy of the electricity load forecasting is crucial in providing better cost effective risk management plans. This paper proposes a Short Term Electricity Load Forecast (STLF) model with a high forecasting accuracy. A cascaded forward BPN neuro-wavelet forecast model is adopted to perform the STLF. The model is composed of several neural networks whose data are processed using a wavelet technique. The data to be used in the model is electricity load historical data. The historical electricity load data is decomposed into several wavelet coefficient using the Discrete wavelet transform (DWT). The wavelet coefficients are used to train the neural networks (NNs) and later, used as the inputs to the NNs for electricity load prediction. The Levenberg-Marquardt (LM) algorithm is selected as the training algorithm for the NNs. To obtain the final forecast, the outputs from the NNs are recombined using the same wavelet technique.
\end{abstract}

Keywords - STLF, BPN and DWT.

\section{INTRODUCTION}

Short term load forecasting (STLF) has taken over a vital role in the operation and planning of power systems since the price depends critically on the accuracy of a load forecast. Forecasting of electricity load demand on half hourly or hourly basis, from one day to many days ahead is referred as Short Term Load Forecasting (STLF).

The aim of STLF is to predict the future electricity load based on the recognition of similar repeating trends of patterns from historical load data [1]. Normally, there are two different methods of forecasting models - the traditional models and the modern techniques. Traditional forecast models entail time series and regression analysis through the use of statistical models such as exponential smoothing, regression, BoxJenkins model, Stste-Space Model, Kalman Filter [2] etc. These models are mostly linear methods and have limited ability to capture non-linearities in the load time series pattern. They require expert knowledge and are much complex to operate.

In the recent years, many researchers switched to try the modern techniques based on artificial intelligence. Of all, the Artificial Neural Network (NN) receives the most attention [3]. NN is regarded as an effective approach and is now commonly used for electricity load forecast. The reason for its popularity is its ease of use and its ability to learn complex input-output relationship. The ability to learn gives NN a better performance in capturing nonlinearities for a time series signal. Therefore, this paper proposes a model comprising neural networks as its forecasting tool.

A NN based forecast model requires historical electricity load data as the input variable to perform STLF. It contain some hidden time-variant pattern. These patterns could be informative to a NN based forecaster to enhance the NN's ability in learning the signals. Thus to extract hidden patterns from the electricity load data, a wavelet decomposition technique is introduced.

\section{Target Electricity Data}

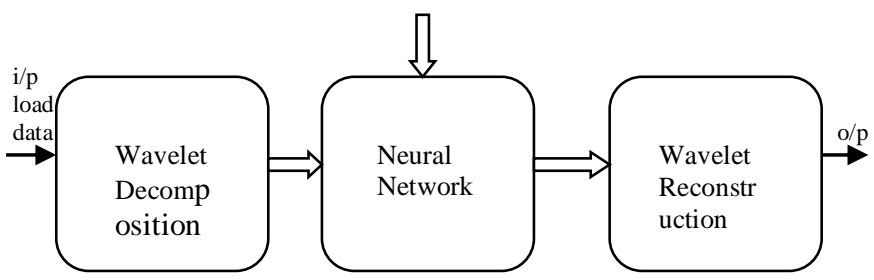

fig.(1)

In the model shown in fig (1), initially wavelet decomposition has been performed on historical electricity load data for one day. Target data for next day also processed as wavelet decomposition. The useful decomposed components of both based on energy level is then feed to the NN model for training, testing and validation. Finally, the output of $\mathrm{NN}$ model is converted into wavelet reconstruction which is the predicted data.

\section{WAVELET TRANSFORM AND NEURAL NETWORK}

In this model, we used wavelet transform and neural network technique. The various methods and models are available. The method/ model which gives better result are discussed here.

\section{A. Wavelet Transform}

Wavelet transform (WT) is a scalable windowing technique. The adjustable window size allows the use of long time intervals when more precise low-frequency information is 


\section{International Journal of Engineering Applied Sciences and Technology, 2021 \\ Vol. 6, Issue 5, ISSN No. 2455-2143, Pages 268-272 \\ Published Online September 2021 in IJEAST (http://www.ijeast.com)}

desired and short time intervals when desiring high-frequency information. Figure (2) illustrates the WT of a time series signal.
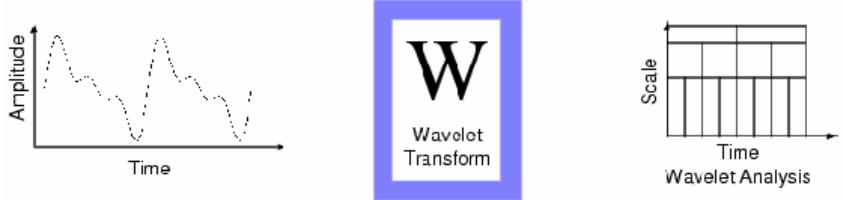

Figure (2): Wavelet Transform of a Time Series Signal

Wavelet transform is of two types- continuous wavelet transform(CWT) and discrete wavelet transform(DWT). It has been observed that DWT gives better result than CWT. Hence in our model we used DWT.

Computation of wavelet coefficients for discrete wavelet transform (DWT) makes use of dyadic scales and positions; that means, scales and positions based on powers of two. The DWT algorithm is capable of producing coefficients of fine scales for capturing high frequency information, and coefficients of coarse scales for capturing low frequency information. If we consider a mother wavelet function and for a given signal, a mathematical representation for a DWT can be expressed as

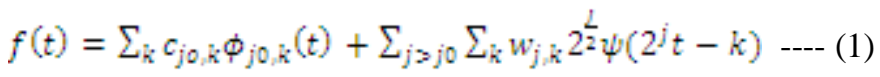

where $\mathrm{j}$ is the dilation or level index, $\mathrm{k}$ is the translation or scaling index, $\phi_{j \mathrm{p} k}$ is a scaling function of coarse scale coefficients $c_{j 0_{\alpha} k}, w_{j k}$ is the scaling function of detail (fine scale) coefficients and all functions of $\psi\left(2^{j} t-\mathbb{k}\right)$ are orthonormal; the summation terms on the left and right of equation (1) represents the approximation and the detail respectively. [4]

Performing DWT, the historical load data has been converted into two parts-approximation coefficient and details coefficient. This process is also referred as wavelet analysis. Approximation coefficient is high scale low frequency component of signal and detail coefficient is low scale high frequency component.

The decomposition perform the convolution between load and high pass/ low pass filter and reconstruction finds the convolution between the load and inverse filter. The decomposition can be implemented using five channels. The reconstructed detail and approximations are true parameters of the original signal as follows[5,6]:

Several wavelet families are available like Haar, Daubechies, Coiflets, Symlet etc. In this paper for the historical load data, DWT has been carried out for Haar,
Daubechies(1 to 10), Coiflets (1 to 5) and Symlet (2 to 8) for various level. At the same time wavelet synthesis also performed and it has been found that the less 'maximum error' is for Daubechies 5 - level 5 family. Hence in the hybrid model the same has been utilised.

\section{B. Neural Network}

The artificial neural network, commonly referred to as neural network $(\mathrm{NN})$, is about a network of interconnected elements. The artificial neuron takes in information through its input lines and the weighted sum of these inputs is obtained by combining the information from all these inputs. An output from the artificial neuron is then generated based on a transfer function which depicts the relationship between the weighted sum of inputs and the output. An example of the transfer function for an artificial neuron can be mathematically expressed as

$$
y=\frac{1}{1+e^{-k x}}
$$

where $y$ is the output of a neuron, $x$ is weighted sum of inputs and $\mathrm{k}$ is the parameter of the neuron.

This transfer function is commonly known as a sigmoid function.

The various NN models are- McCulloch-Pitts Neuron, The Perceptron, FFBPN, CFBPN, MLP, SVM ANN etc. [7,8 In our hubrid model, CFBPN model is used with LM training algorithm. The wavelet decomposed data has been given to CFBPN as an input. Amongst the available data $70 \%$ of input data is used for training the model, $15 \%$ is for testing and $15 \%$ for validation.

\section{HYBRID NEURO WAVELET MODEL}

The proposed hybrid neurowavelet model has three main stages as shown in fig (3).

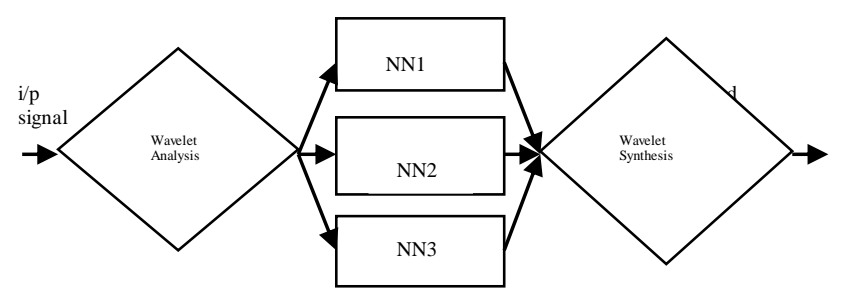

Fig.(3)

As shown in Figure 3, wavelet analysis has been implemented in the first stage and wavelet synthesis at the last stages. The actual historical electricity load data is first decomposed into five wavelet detail coefficient signals and one approximation signal. The decomposed signals are then applied as an input to the NNs at the second stage to predict the future load demand patterns for each of the signals. Finally, the predicted signals 
are recombined in the third stage to form the final predicted load data.

\section{A. Pre-Signal Processing}

For pre signal processing, historical electricity load data is given to the model where Discrete Wavelet Transform (DWT) is used as the pre signal processor. Here the selected resolution level is five of Daubechies 5 family, hence the respective electricity load data is decomposed into five wavelet detail coefficients and one approximation coefficient. These decomposed coefficients are then applied as an inputs to the predictor model (NNs) for either training or forecasting use. The decomposition process is illustrated in Figure 4.
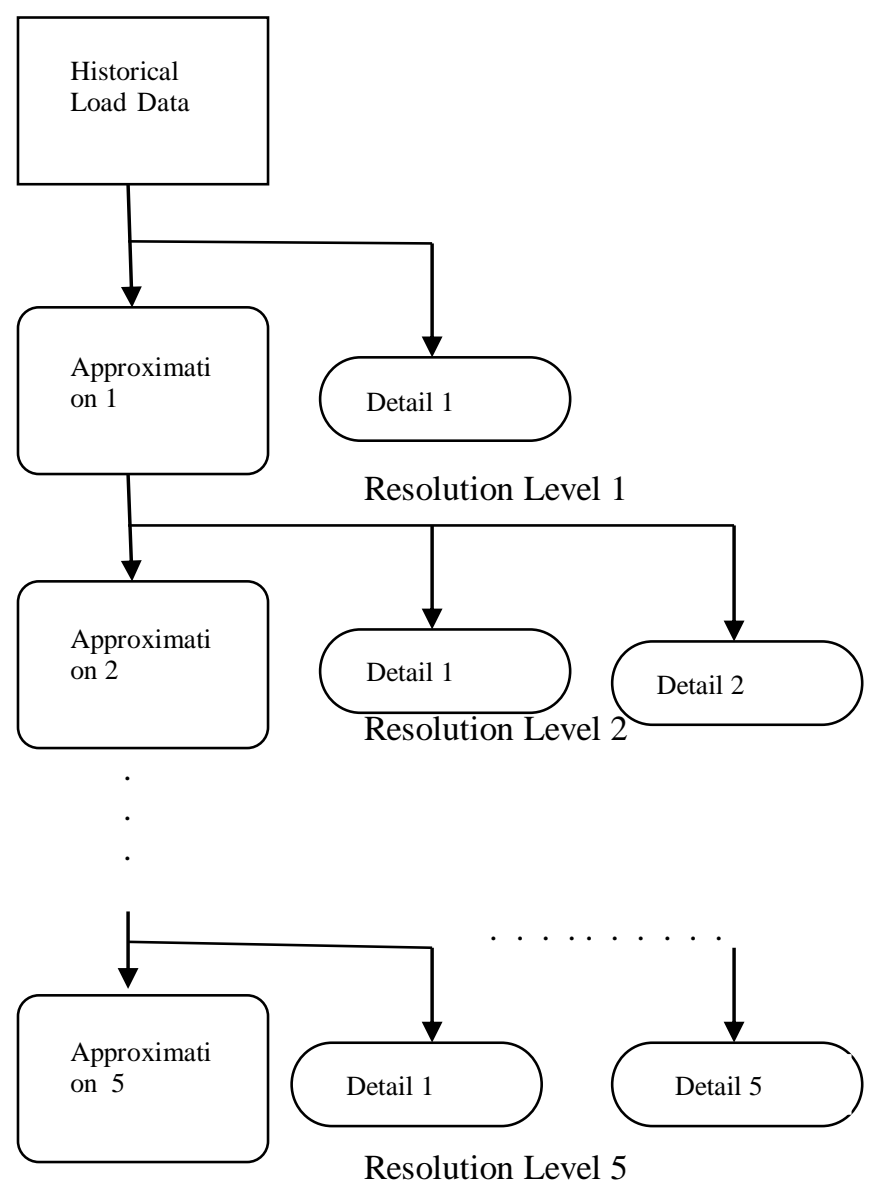

Fig.(4) Wavelet Decomposition Process

The most suitable resolution level is identified based on the smoothness of the approximation signal at specified level. The desired approximation signal should depict a general pattern of its original. A higher resolution level would produce a smoother approximation signal.

\section{B. Signal Prediction}

NNs are used for prediction of electricity load demand in the forecast model. The number of NNs needed for the model is determined by the number of wavelet coefficient signals at the output of the pre-processor. For each wavelet coefficient signal (including the approximation), one $\mathrm{NN}$ is required to perform the corresponding prediction. In proposed model six NNs are used.

\section{C.Post Signal Processing}

For post signal processing, the same wavelet technique (DWT) and resolution level as mentioned in section A are used. In this stage, the outputs from the proposed hybrid forecasting model (NNs) are combined to form the final predicted output. This is achieved by summing all the predicted wavelet coefficients. Figure 5 illustrates the recombination process.

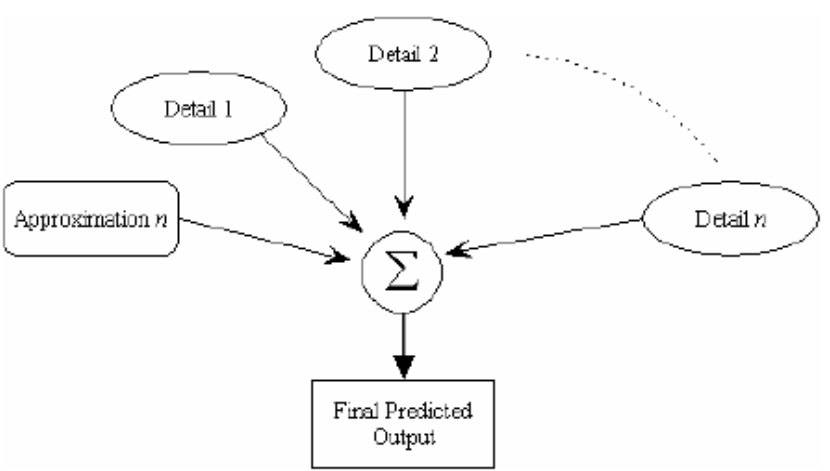

Fig.5: Wavelet Recombination Process

\section{D.Finalizing the Design}

Cascaded feedforward backpropagation network (CFBPN) algorithm is used in the proposed forecast model since it gives better result than the popular BPN network.

\section{E. Number of Input Nodes and Output Neurons}

The nodes in the input layer are only used to distribute input variables to the hidden layer. As mentioned earlier, the training electricity load data is taken Therefore, the total number of input nodes for the proposed model is fixed at six. The forecast model is set to have one output neuron that takes one value of the electricity data as the target.

\section{F. Number of Hidden Neurons}

The learning capability of a NN depends on the number of neurons in its hidden layer. Determining the number of hidden neurons is important; if the number of hidden neurons is insufficient, the consequence will result in an inaccurate approximation. On the other, excessive hidden neurons are not desirable. This will affect the learning progress of the network. In other words, instead of learning the problem, the network will tend to memorize it. Over-sizing the hidden layer can also lead to longer training time. With adequate time for 
testing, the most suitable number of neurons for the $\mathrm{NN}$ is explored.

\section{G. Learning Algorithm}

As mentioned earlier, the CFBPN algorithm will be used as the learning algorithm for the forecast model. Using this algorithm will significantly shorten the time needed to train the NNs. Further information regarding CFBPN algorithm and its benchmark with other algorithms is obtainable from the MATLAB® help file.

\section{H. Final Proposed Forecast Model}

After finalizing the design considerations, the final proposed $\mathrm{NN}$ forecast model is as depicted in Figure 6. The model is a two-layer feed-forward BP NN with two layers of adjustable weights.

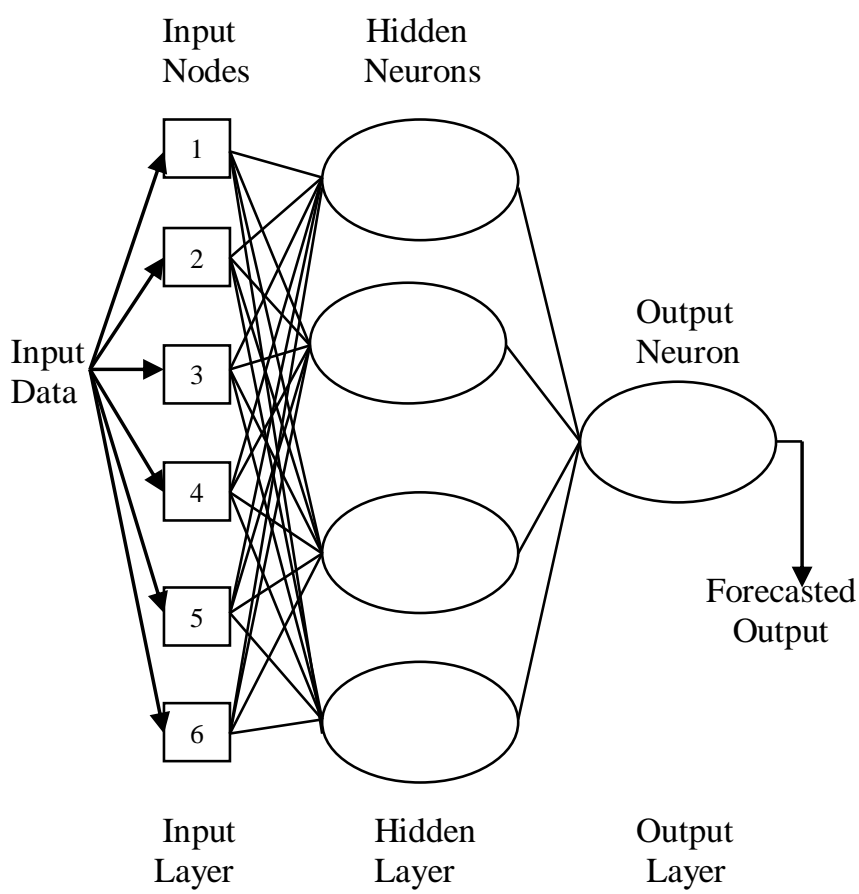

Fig.6: Final Proposed NN Forecast Model

\section{SIMULATION RESULTS}

The proposed hybrid neuro wavelet model is designed in Matlab2009a.

The proposed model is tested with 48 data points of historical data containing the electricity load for the month of January 2010, on a half-hourly basis of Queensland Electricity Board. Before the wavelet decomposition technique (DWT) is applied, the sets of Electricity load data is first normalized and then multiplied by a power consumption factor.

The model is evaluated based on it prediction errors. A successful model would yield an accurate forecast. The performance of the model is measured using the mean square error (MSE), MSE is expressed as -

$$
\mathrm{MSE}=\frac{1}{W} \sum_{i=1}^{W}\left[\sum_{j=1}^{y_{j}}\left(t_{i j}-y_{i j}\right) 2\right.
$$

Where $t_{\mathrm{ij}}$ is desired output and $\mathrm{y}_{\mathrm{ij}}$ is actual output[9] Mean Square Error found is $2.07 \times 10^{-3}$

The wavelet decomposed electricity load data is as shown below in fig. 7
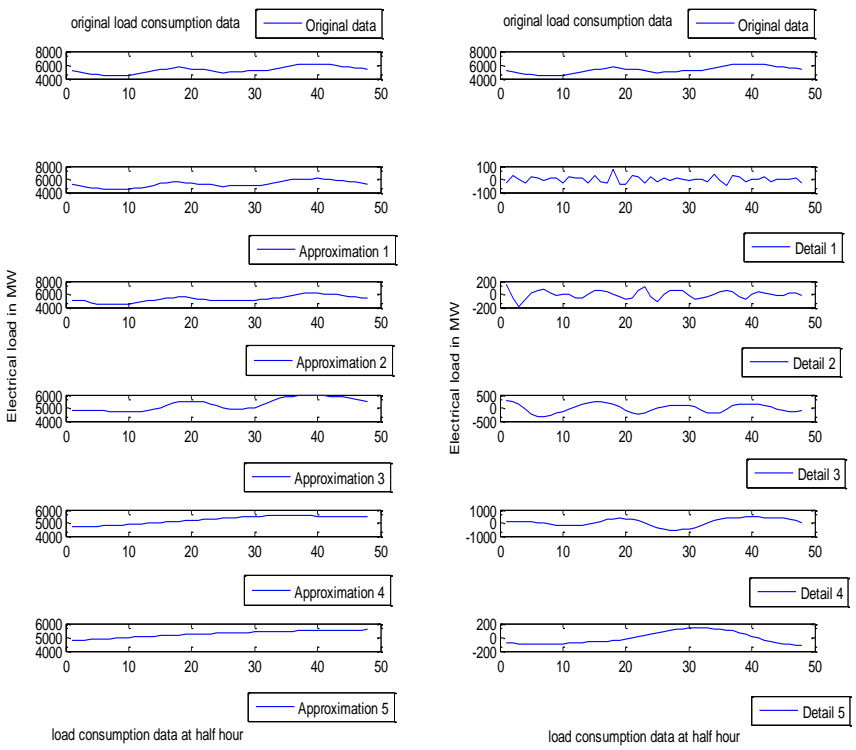

fig.7:Wavelet decomposed electricity load data

The wavelet decomposed electricity target data is as shown below in fig. 8
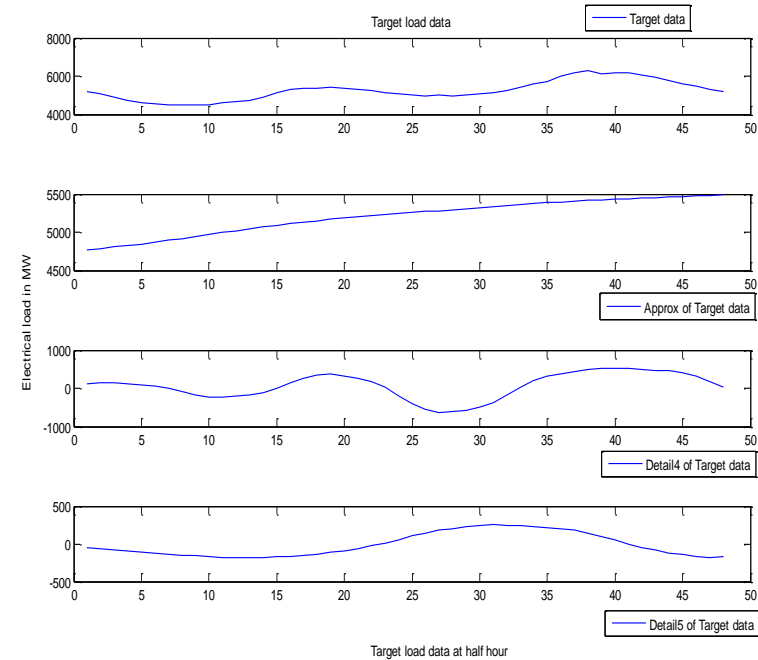

fig.8: Wavelet decomposed electricity target data 


\section{International Journal of Engineering Applied Sciences and Technology, 2021 \\ Vol. 6, Issue 5, ISSN No. 2455-2143, Pages 268-272 \\ Published Online September 2021 in IJEAST (http://www.ijeast.com)}

The actual output and forecasted output is as shown in fig.9
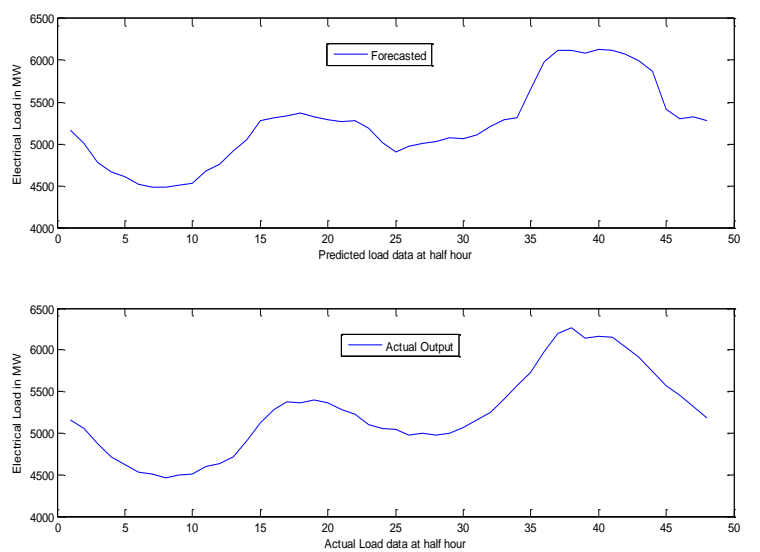

fig.9:Actual \& Predicted output

The energy content of Approximation and Detail Coefficient of Input data and target data is as shown in table 1

\begin{tabular}{|l|l|l|}
\hline Particulars & Input Data & Target Data \\
\hline Approx.5 Coefficient & 99.814 & 99.7637 \\
\hline Detail 1 Coefficient & 0.000377 & 0.000198 \\
\hline Detail 2 Coefficient & 0.002751 & 0.001292 \\
\hline Detail 3 Coefficient & 0.031110 & 0.030872 \\
\hline Detail 4 Coefficient & 0.128360 & 0.159320 \\
\hline Detail 5 Coefficient & 0.023404 & 0.044597 \\
\hline \multicolumn{2}{|l}{ Table.1 }
\end{tabular}

Target data ,Projected data and corresponding error for first eight data points is as shown in table 2

\begin{tabular}{|l|l|l|}
\hline Target Data & Predicted Data & Error \\
\hline 5164.02 & 5167.33 & -3.31 \\
\hline 5053.05 & 5052.13 & 0.92 \\
\hline 4878.52 & 4877.12 & 1.4 \\
\hline 4709.31 & 4709.01 & 0.3 \\
\hline 4619.11 & 4619.04 & 0.07 \\
\hline 4530.67 & 4531.45 & -0.78 \\
\hline 4513.66 & 4512.12 & 1.54 \\
\hline 4466.61 & 4467.08 & -0.47 \\
\hline
\end{tabular}

\section{CONCLUSIONS}

This paper proposed a STLF model with a high forecasting accuracy. The DWT has been successfully implemented in the model. The implementation of DWT has reasonably enhanced the learning capability of the NNs in the model, thus minimizing their training frequencies as shown in the simulations.

In summary, the inclusion of electricity load data and the use of DWT (as the data processing tool) for the proposed STLF model have been a success.

\section{REFERENCES}

[1] T. Down, "Introduction to Neural Computing \& Pattern Recognition," COMP3700 Machine Learning website, University of Queensland, Australia, http://www.csee.uq.edu.au/ comp3700/lectures/lecture1. pdf (current Jul. 2002)

[2] Slobodan A Ilic, S.M.Vukmirovic, A.M.Erdeljan and F.J.Kulic," Hybrid artificial neural network system for short term load forecasting," Thermal Science, Year 2012, Vol.16, Suppl.1, pp. S215-S224.

[3] H. S. Hippert , "Neural Networks for Short-Term Load Forecasting: A Review and Evaluation," IEEE Trans. On Power Systems, Vol. 16, No. 1, Feb. 2001, pp. 44-54

[4] Ismail B, Ataulla and Mohammed Yunus ,'Time Series Forecasting using undecimated wavelets,neural networks and genetic algorithm," International Journal of Electronics and Computer Science Engineering ISSN 2277-1956/V1N3-1404-1415

[5] P.Bunnoon,K.Chalermyanont and C.Limsakul,"Wavelet and neural network approach to demand forecasting based on whole and electric sub-control center area," International Journal of Soft Computing and Engineering ISSN:2231-2307,Volume-1,Issue-6,January 2012

[6] A.S.Pandey,Devender Singh and S.K.Sinha,'Intelligent hybrid wavelet models for short term load forecasting," IEEE Trans. On power system, vol.25,pp 12661273,2010

[7] M.Moazzami,A.Khodabakhshian and R.Hooshmand,"A New hybrid day ahead peak load forecasting method for Iran's national grid," www.elsevier.com/locate/apenergy

[8] R.Barzamini, F.Hajati,S.Gheisari and M.B.Motamadinejad,"Short term load forecasting using multilayer perceptron and fuzzy inference systems for Islamic countries," Journal of Applied Sciences 12(1):40-47,2012 ISSN 1812-5654

[9] Hongsheng Su,'Chaos quantum-behaved particle swarm optimization based neural networks for short term load forecasting,'Procedia Engineering 15 (2011) 199-203 